\title{
THE USE OF PHASE DIAGRAMS IN DISSOLUTION STUDIES
}

\author{
by
}

A. R. Cooper

Department of Metallurgy

Case Western Reserve University

Cleveland, Ohio 44106

$$
N Q R-36-003-100
$$

Introduction

Many important phenomena in metallurgy, ceramics, and geology depend on dissolution. Corrosion of refractories by molten slags and glasses, as well as the erosion of the earth's crust by rivers and streams, is affected by the kinetics of dissolution. The rate of melting of glass is often limited by the rate of dissolving sand grains, while the rate of refining a glass melt is similarly determined by the kinetics of dissolution of gas bubbles. The rate of "essentially" solid state reactions is enhanced by the presence of a liquid which permits a dissolution-reprecipitation process to provide an easy path for the reaction. For example, the presence of a small amount of molten calcium silicate accelerates the transformation of quartz to cristobalite in the firing of silica brick.
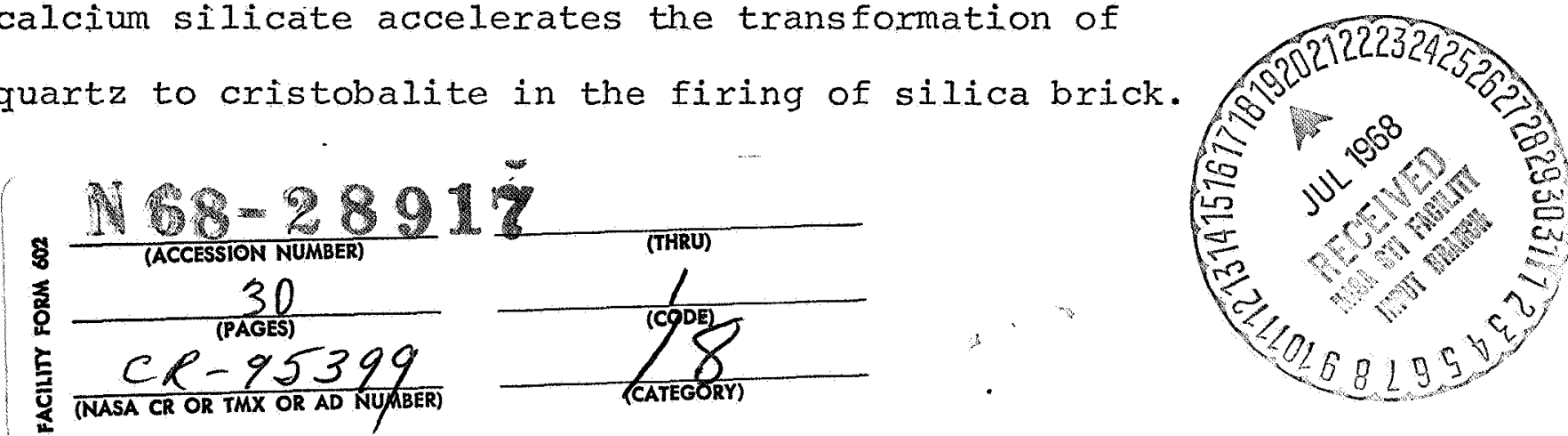
Mention of dissolution-reprecipitation phenomena calls attention to the sometimes overlooked consideration that precipitation and dissolution are the same process in opposite direction. This leads us to define dissolution and precipitation by the following equation:

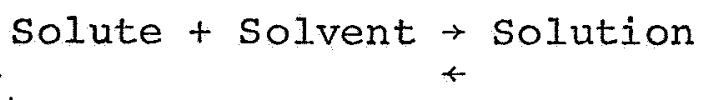

When reaction goes to the right, it is dissolution, and when to the left, it is precipitation.

Although in the following we will invariably be considering the solute to be solid and the solvent and solution to be liquids, there is no restriction as to the phase of any of the components. (However, "dissolution" of a liquid by a gas is more commonly called "evaporation").

\section{Mechanism for Dissolution}

The classic work in the field of dissolution kinetics is that of Noyes and whitney (1) who, in 1897, rotated cylinders of lead chloride in benzoic acid and water; noted the change of concentration of lead chloride in the solution, and speculated that the rate of dissolution was controlled by the rate at 
which atoms diffused out from the solute interface.

Several years later, Nernst ${ }^{(2)}$ made this concept explicit by postulating that chemical reaction takes place so rapidly at the solvent interface that the solution is quickly saturated there and remains so during the dissolution process. Furthermore, he presumed that beyond a certain distance, $\delta$, the concentration is that of the bulk solution, $\mathrm{C}_{\infty}$. This allows the following expression (since called the Noyes-Nernst Equation) to be written for the flux density, $j$, across the solute interface - of area, A, and for the change in bulk concentration, $c_{\infty}$, with time, $t$, for a solution of volume, $v$ :

$$
V \frac{d C_{\infty}}{d t}=j A=\frac{D}{\delta}\left(C_{\text {sat }}-C_{\infty}\right) A-\left(C_{\text {sat }} C_{\infty}\right) / \nabla C_{\text {interface }}
$$

where $C_{\text {sat }}$ is saturation concentration, D is diffusion coefficient, and $\delta^{*}=\frac{-\left(C_{\text {sat }}-C_{\infty}\right)}{(\nabla C)_{\text {interface }}}$, as shown by the schematic diagram in Fig. 1. Although at high values of $\mathrm{C}_{\text {sat' }} \mathrm{Eq}$. (1) needs correction ${ }^{(3)}$ to account for the effect of the moving interface, it has been extremely useful as it stands in organizing large amounts of data on dissolution kinetics in widely varying systems. 
As Berthoud ${ }^{(4)}$ showed in 1912 , it is relatively easy to generalize a bit further and permit a finite value of a surface reaction rate constant, $K$, which leads to:

$$
j=\left\{\frac{K}{1+(K \delta / D)}\right\} \quad\left(C_{\text {sat }}-C_{\infty}\right)
$$

The existence of Eqs. (1) and (2) allowed studies of dissolution kinetics to proceed in a systematic way for the past half century. (5) Furthermore, the development of boundary layer theory ${ }^{(6)}$ during this period has permitted the empirical constant, $\delta$, to be derived from first principles for a variety of cases of forced and free convection. For experimental studies of dissolution, one of the most useful consequences of boundary layer theory is the fact that the effective boundary layer thickness, $\delta$, at the surface of a dissolving rotating disc is independent of radius and proportional to the square root of angular velocity. (7) Thus, rotation over a wide range of velocities permits determination of $D$ and $K$, provided $\frac{K}{D}$ is not always $>\frac{1}{\frac{1}{\delta}}$. There remain situations of interest where values for ${ }^{*}$ have not been calculated. Noteworthy are cases of free convection where the flow is driven not by 
density differences between bulk and saturated solution, but rather by surface energy differences. (8) Also the relationship of the diffusion coefficient, D, for dissolution, to the mobility of the individual species in the solution, is not entirely resolved; however, the appropriateness of using a single diffusion coefficient, even if the system is multicomponent, has been verified theoretically ${ }^{(9)}$ and experimentally ${ }^{(10)}$ for cases where $C_{\infty}$ remains relatively unchanged.

Eqs. (1) and (2) both permit understanding of the rate of dissolution as the product of two factors - a potential difference, measured by $\left(\mathrm{C}_{\mathrm{Sat}}-\mathrm{C}_{\infty}\right)$, and a reciprocal resistance, measured by $\left\{\frac{\mathrm{K}}{1+(\mathrm{K} \delta / \mathrm{D})}\right\}$.

To make graphic the concept of a boundary layer thickness, we showed in Fig. I the concentration distribution in the vicinity of an interface. Let us now consider ${ }^{*}$ to be fixed (by boundary layer theory), and inquire about the differences in concentration profile for three distinct cases of dissolution kinetics:

(1) transport control: $K>D / \delta^{*} ; j=D / \delta^{*}\left(C_{\text {sat }}-C_{\infty}\right)$

(2) mixed control: $\mathrm{K} \simeq \mathrm{D} / \delta_{\delta}^{*} ; \quad(\mathrm{Eq} \cdot(2)$ applies)

(3) phase boundary control: $\mathrm{K} \ll \mathrm{D} / \delta_{i}^{*} \mathrm{j}=\mathrm{K}\left(\mathrm{C}_{\text {sat }}-\mathrm{C}_{\infty}\right)$ 
Fig. 2 shows, schematically, the concentration distribution for each case. Note that in mixed control, the potential difference, $\left(C_{\text {sat }}-C_{\infty}\right)$, is divided into two parts. One, $\left(\mathrm{C}_{\text {sat }}-\mathrm{C}_{\text {int }}\right)$, drives the phase boundary reaction, and the other, $\left(C_{\text {int }}-C_{\infty}\right)$, drives the transport process. The potential difference divides so as to keep the dissolution rates from each process equal, i. e.:

$$
\mathrm{K}\left(\mathrm{C}_{\text {sat }}-\mathrm{C}_{\text {int }}\right)=\mathrm{D} / \hat{\delta}^{*}\left(\mathrm{C}_{\text {int }}-\mathrm{C}_{\infty}\right)=\mathrm{j}
$$

This gives a so-called "stationary non-equilibrium" state for which the rate of entropy production is a minimum. (11)

When $\delta^{*}$ is allowed to change $\left(\delta^{*} \sim t^{1 / 2}\right.$ when convection is absent), we may have a case where at short times $\mathrm{D} / \delta$. $>\mathrm{K}$, at moderate times $\mathrm{D} / \delta_{\delta}^{*} \simeq \mathrm{K}$, and at long times $D / \delta^{*} \ll K$. For this situation the process would change from interface control to mixed control to transport control as time advanced. Similarly, the interface concentration, $C_{\text {int }}$, would increase from $\mathrm{C}_{\infty}$ toward $\mathrm{C}_{\mathrm{sat}}$ as time advanced. We recognize that although the thermodynamics of a system do affect D, particularly through corrections associated with non-ideal behavior in the solution, the primary manifestation of the thermodynamjes is through the potential $\left(-C_{\text {sat }}{ }^{-} \mathrm{C}_{\infty}\right)$. Further, 
since the bulk concentration, $\mathrm{C}_{\infty}$, is given to us or chosen by us, the only quantity entirely established by thermodynamics is the saturation concentration, $C_{\text {sat' }}$ and this is what we expect to find from the appropriate phase diagram.

\section{Binary Solutions}

In a binary solution, the saturation composition at a given temperature can be expressed by a single number, $C_{\text {sat' }}$ which is readily determined if a phase diagram is available. This is shown schematically in Fig. 3, the $\mathrm{SiO}_{2}$ rich portion of the $\mathrm{Rb}_{2} \mathrm{O}-\mathrm{SiO}_{2}$ phase diagram. (12)

The liquidus line gives the mol fraction at saturation as a function of temperature, $\mathrm{x}_{\text {sat }}(\mathrm{T})$. To obtain molar concentration, $C$, from mol fraction, $\mathrm{x}$, we must multiply by the molar density, $\rho$, which for silica-rich rubidium silicate $/$ is about $0.040 \mathrm{mols} / \mathrm{cc}$. As an example, we note that the saturation concentration of silica at $1500^{\circ}$ is given by:

$$
C_{\text {sat }}\left(1500^{\circ}\right) \simeq 0.95 \times 0.040=.038
$$

The concentration of silica in the bulk, $\mathrm{C}_{\infty}$, of course, may be any value. Consider the case where the mol fraction in the bulk, $x_{\infty}=0.90\left(c_{\infty} \simeq 0.0360\right)$, 
as indicated by the dotted line on Fig. 3. We note that $\left(C_{\text {sat }}-C_{\infty}\right)$ has its maximum of about 0.0040 mols $/ \mathrm{cm}^{3}$ at $1713^{\circ} \mathrm{C}$, the melting point of cristobalite. The value of $\left(C_{\text {sat }}-C_{\infty}\right)$ decreases with cooling until it reaches zero at about $1280^{\circ} \mathrm{C}$ where $\mathrm{X}_{\text {sat }}=.90$. Below this temperature $\left(\mathrm{C}_{\mathrm{sat}}{ }^{-}\right.$ $\mathrm{C}_{\infty}$ l becomes negative which reveals that from $1280^{\circ} \mathrm{C}$ downward, precipitation rather than dissolution of silica will occur.

It pays to examine this point a bit more closely using Eq. (2). Rate constants for chemical reactions typically have an Arrhenius-type temperature dependence, i.e., $K=B$ exp $(A / T)$. Similarly, the temperature dependence* of $D / \delta$ can usually be represented by $D / \stackrel{*}{\delta}=F$ exp $-G / T$. FrequentIy, $\mathrm{K} \gg \mathrm{D} / \AA$ or vice versa. Then, the entire reciprocal resistance to the dissolution process (expression in brackets in Eq. (2) $l$ is likewise given by an Arrhenius relation, and Eq. (2) can be written:

$$
j=\{L \exp -(M / T)\}\left(C_{S}-C_{\infty}\right)
$$

"The validity of this statement is verified from the following considerations: i-viscosity and diffusivity in liquids show Arrhenius behavior over wide temperature intervals, ii-the effective boundary layer thickness, $\delta$, for laminar free or forced convection acquires its temperature dependence from its proportionality to fractional powers of viscosity and diffusivity (5d), ili-when convection is unimportant, the temperature dependence of $\delta$ arises solely through its proportionality to a fractional power of $\mathrm{D}$ (5d). 
The information in Fig. 3 plus Eq. (3) allows us to calculate $j / L$ (I can be considered just a scale factor) as a function of reciprocal temperature, $\frac{1}{T}$. Then, as is common practice, we present the data by plotting $\ln (j / L)$ vs. $\frac{1}{T}$. Fig. 4 shows such a plot for the case where $M=10^{4}\left({ }^{\circ} \mathrm{K}\right)^{-1}$, equivalent to an activation energy, $M R$, of $R \times 10^{4}$ cal per mol where $R$ is the gas constant, $\simeq 2 \mathrm{cal} / \mathrm{mol}^{\circ} \mathrm{K}$. As the liquidus temperature, $\mathrm{T}_{L}$, is approached from above, the "apparent activation energy", given by the negative of the slope in Fig. 4, begins to increase rapidly. This is a manifestation of the fact the temperature dependence of $\left(c_{s}-C_{\infty}\right)$ is not exponentially related to reciprocal temperature. Therefore, Fig. 4 is an example of an inappropriate attempt to force an Arrhenius relation onto the overall dissolution process.

If data close to the liquidus temperature is obtained, the fallacy is evidenced by the sharp curvature in the $\log (j / D)$ vs. $\frac{1}{T}$ plot. However, if data is only obtained in the region decidedly above the liquidus temperature (e. $g ., T>\frac{10^{4}}{6}$ ), then an "apparent activation energy" $\left(\frac{2 \cdot 3}{R}\left(d \log (j / L) / d\left(\frac{1}{T}\right)\right)\right.$ is obtained of about $2 \mathrm{R} \times 10^{4} \mathrm{cal}$. That the "apparent activation energy" of approximately $2 \mathrm{R} \times 10^{4} \mathrm{cal} / \mathrm{mol}{ }^{\circ} \mathrm{K}$ is in disagreement with the true value of $\mathrm{MR}=\mathrm{R} \times 10^{4} \mathrm{cal} / \mathrm{mol}{ }^{\circ} \mathrm{K}$ shows 
that plots of log rate of solution versus reciprocal temperature may indeed give poor estimates of the true activation energy for the kinetic factors (reciprocal resistance) that affect dissolution. Mention should be made that dissolution can also occur into a metastable solvent. We examine this possibility briefly by using Figure 3 again. Consider the composition, $x_{\infty}=0.80$, as solvent. Beneath $1160^{\circ} \mathrm{C}$, precipitation of $\mathrm{Rb}_{2} \mathrm{O} \cdot 2 \mathrm{SiO}_{2}$ can occur. However, if precipitation of $\mathrm{Rb}_{2} \mathrm{O} \cdot 2 \mathrm{SiO}_{2}$ is a slow process, dissolution may occur with its rate correctly predicted by Eq. (2). Even beneath the eutectic temperature of $870^{\circ} \mathrm{C}$, dissolution of $\mathrm{SiO}_{2}$ can still occur, provided it is a much more rapid process than precipitation of $\mathrm{Rb}_{2} \mathrm{O} \cdot 2 \mathrm{SiO}_{2}$. To use Eq. (2), it is necessary to extend the liquidus line into this two-crystal region, as shown by a dashed line on Fig. 3. Note that as cooling occurs, the potential difference for precipitation of $\mathrm{Rb}_{2} \mathrm{O} \cdot 2 \mathrm{SiO}_{2}$ increases while the potential difference for dissolution decreases until it vanishes at some temperature where the extended metastable liquidus line intersects the bulk composition, $\mathrm{x}=.080$. At this temperature the dissolution process must cease. 
By determining the driving force for dissolution from phase diagrams, we can rank* the suitability of different refractories to contain specific melts. Naturally, to minimize the rate of solution, we wish to minimize $\left(C_{\text {sat }}-C_{\infty}\right)$, that is $\left(x_{\text {sat }}-x_{\infty}\right)$. Thus we want to have $\mathrm{x}_{\text {sat }}$ as low as possible at a given temperature. Using the collection, Phase Diagrams for Ceramists, (13), we see, for example, that in the zirconium oxide-silica system the value, $\mathrm{x}_{\text {sat }}\left(\mathrm{z}_{\mathrm{r}} \mathrm{O}_{2}\right)$, at $1800^{\circ} \mathrm{C}$ is much less than the value for $\mathrm{x}_{\text {sat }}\left(\mathrm{Al}_{2} \mathrm{O}_{3}\right)$ in the alumnia-silica system at $1800^{\circ} \mathrm{C}$. Hence, zirconium oxide appears superior for containing high temperature silicate melts, a fact already well verified industrially. From similar considerations alumina seems well suited for containing borate melts beneath $1600^{\circ} \mathrm{C}$.

When there are no phase diagrams for a system of interest, some estimate of solubility can be obtained by using the van't Hoff equation. For ideal solutions:

$$
\frac{\mathrm{d} \ln \mathrm{X}_{\text {sat }}}{\mathrm{dT}} \simeq \frac{\Delta \mathrm{H}_{\mathrm{f}}}{R \mathrm{~T}^{2}}
$$

where $\Delta \mathrm{H}_{f}$ is the heat of fusion per mol. Integration from

\footnotetext{
This estimate ignores the kinetic factors, i.e., the reciprocal resistance, (in brackets in equation 2l and as such must be used cauteously unless some knowledge of atomic mobilities exists.
} 
the melting point, $T_{m}$, to a specific temperature, $T$, yields

$$
\text { In } \left.x_{\text {sat }}=\int_{T_{m}}^{T} \frac{\Delta H_{f}}{R T^{2}} d t=\frac{\Delta H_{f}}{R} \cdot I_{\frac{m}{T_{m}}-T}^{T}\right\}
$$

For low potential for dissolution, we desire low values of $\mathrm{x}_{\text {sat }}$. Therefore, we look for materials with high melting points and high values of heat of fusion. For non ideal solutions high values of heat of mixing also give reduced solutiliby, $x_{\text {sat }}$.

We have mentioned the value of phase diagrams to dissolution studies in binary systems. The converse value should also be mentioned. Plots of $j$ vs. $T$ are perhaps the most accurate means of determining liquidus temperature. As the liquidus temperature, ${ }{ }_{L}$ ' is approached, the rate of dissolution becomes nearly proportional to $\left(T-T_{L}\right)$. Extrapolation, or better still, interpolation to $j=0$ gives a good estimate of $\mathrm{T}_{L}$, although a word of caution should be mentioned. While the rate, $j(T)$, must be continuous as $T$ passes through $\mathrm{T}_{\mathrm{L}^{\prime}}$ it is possible that the temperature dependence, $d j / d T$, is discontinuous at $T=T_{L}$. For example, in some systems, the interface rate constant, $\mathrm{K}_{\mathrm{dis}}$, for dissolution may be very high (disordering from a crystal to a liquid structure is an easy process) and $K_{d i s} \gg D / \delta$ at $\mathrm{T}^{*} \rightarrow \mathrm{T}_{\mathrm{L}}{ }^{+}$. However, the interface rate constant, $\mathrm{K}_{\mathrm{ppt}}$, for 
precipitation may be very small, (rearranging from the liquid to the precise crystalline arrangement may be much more difficult than disordering) and $\mathrm{K}_{\text {ppt }} \gg \mathrm{D} / \delta^{*}$ at $\mathrm{T} \rightarrow \mathrm{T}_{\mathrm{L}}^{-}$. For this case, we might expect $j(T)$ to look schematically as shown in Fig. 5. Notice that for this case interpolation between $\left(T_{L}+10\right)$ and $\left(T_{L}-10\right)$ can give a marked error.

\section{Multicomponent Systems}

When, as in the case in most practical dissolution problems, the reaction of solvent and solute leads to a multicomponent system, the analysis becomes more complicated. For an " $n$ " component system the phenomenological treatment of multicomponent diffusion requires (14) , that the coefficient of equation 2 be replaced by a rwo matrix of (n - 1) coefficients ${ }^{(14)}$. No longer is the chemical composition defined by a single concentration, nor is there a single saturation composition at a given temperature. Instead, (n - 1) concentrations are required to describe the composition, and a connected set of compositions is needed to form an isothermal liquidus surface. $\mathbb{P}_{\text {In a ternary system, the isothermal }}$ liquidus surface is a line which, in the absence of compound formation may look as shown in Figure 6 for the system $\alpha-\beta-\gamma$. The diffusion path (i.e., the continuous collection of compositions between the solvent and the interfacel on Figure 6 is appropriate for the dissolution of pure solid $\alpha$ by a melt 
"S" for the case where $\beta$ is more mobile than $\gamma$ and dissolution is transport controlled.

Consistent with usual behavior (15), the diffusion path shown in Figure 6 does not follow the straight line $\overline{\alpha S}$ between the solute, $\alpha$, and solvent, $S$, but exhibits curvature. The course of this path is determined by several considerations.

1. One end point is at solvent composition, $\mathrm{S}$.

2. If the dissolution is transport-controlled, the other end point is on the. liquidus line, $l$; otherwise, it lies between $\ell$ and $S$.

3. The average ratio of the concentration of $\beta$ to $\gamma,\left(C_{\beta} / C_{\gamma}\right)$, within the solution is equal to this ratio in the original bulk solvent.

4. Within the restraints of 1,2 , and 3 , the path is chosen so as to give the minimum resistance to dissolution*.

Let us examine points 3 and 4 above more closely. "3", is derived from conservation of $\beta$ and $\gamma$. Each incremental volume of the solution has a composition which lies on the diffusion path. Thus, the composition is a function of position in the solution. Following the direction of the concentration gradient from solute to solvent, we plot on Fig. 7 the

"In the language of irreversible thermodynamics, this is the "stationary path" which leads to minimum entropy production. 
concentration, $\mathrm{C}_{\beta}, \mathrm{C}_{\gamma}$, and their ratio, $\mathrm{C}_{\beta} / \mathrm{C}_{\gamma}$ as a function of volume from the interface* . Fig. 7 is consistent with Fig. 6 in the sense that the continuous collection of composition points on Fig. 6 have ratios, $C_{\beta} / C_{\gamma}$, which are identical to the collection of ratios obtained on Fig. 7. "3" now can be stated explicitly as:

$$
\left.v_{\Sigma}^{-1} \int_{v_{\Sigma}}\left(c_{\beta} / c_{\gamma}\right) d v=\frac{c_{\beta}}{c_{\gamma}}\right)_{\text {solvent }}
$$

where $V_{\Sigma}$ is the total volume of the solution.

Equation 4 applies to Fig. 7. Hence, in no sense does it require that, on Fig. 6 , the area $Q_{1}$ below the diffusion path and above line $\overline{a S}$ equal the area, $Q_{2}$, below the diffusion path and above $\overline{\alpha S}$. It does, however, require that the diffusion path to intersect $\overline{\alpha S}$ on Fig. 6 .

To consider "4" we note that the diffusion path of least resistance to dissolution is determined by the mobilities and thermodynamic activities of the individual components. Figs. 6 and 7 illustrate a case where the dominant influence stems from the relatively higher mobility of one species, $\beta$, compared to the other, $\gamma$.

An intuituve understanding of the course of the diffusion

\footnotetext{
"If $\Delta r$ is linear distance from interface, the volume from the interface, $V$ is given by $V=\Delta R R^{2}$ for a spherically symetric solute, $\mathrm{V} \cong \triangle \mathrm{RR} \hat{\mathrm{H}} \mathrm{H}$ for a long cylindrical solute of length $H$ and $V \simeq \triangle R A$ for a large slab of area, $A$.
} 
path begins with the recognition that dissolution of $\alpha$ requires both $\beta$ and $\gamma$ to diffuse to the interface. For our purpose, we do not require use of the formalism of multicomponent diffusion (14). Rather, it is sufficient to appreciate that the product of the gradient of a species and its mobility will exert a major influence on its flux density ${ }^{(16)}$. Because of its higher mobility, $\beta$ experiences less resistance - in diffusing to the interface and thus it is relatively more abundant at the interface than the less mobile species, $r$. Furthermore, the mobile species, $\beta$, requires a less steep concentration gradient than species $\gamma$ to maintain equilibrium at the solvent interface. Finally, the more mobile species, $\beta$, can diffuse over longer distances. Hence, at long distances fron the interface, $\beta$ will deviate nore from the solvent concentration than will $\gamma$.

All of these deductions are consistent with what is shown in Figures 6 and 7 and more important, what has been found experimentally in the dissolution of silica glass and sapphire in molten CaO $\cdot \mathrm{Al}_{2} \cdot \mathrm{SiO}_{2}{ }^{(17)}$.

Provided the bulk composition, $s$, does not change, the principles of irreversible thermodynamics dictate that the diffusion path will remain fixed because this is the path of minimum entropy production and any fluctuation fron it will be unistable $(11)$.

An importance of this fixed path is that it permits the 
use of equation 2 directly for dissolution in multicomponent systems; concentrations now refers to concentration of solute, $\alpha$, and the diffusion coefficient refers to the effectice binary diffusion coefficient, $\operatorname{EBDC}(\alpha)$, for the solvent in the multicomponent system ${ }^{(9)}$.

Additional complications can occur during dissolution in multicomponent systems. Worthy of note is a process termed "Incongruent Dissolution" by Oishi, et al. (17) and described as the process whereby a "solid converts by dissolution to a liquid and another solid". It is similar to the case described earlier of dissolution by a super-cooled solvent in a binary system. However, in the ternary case, the solvent may be a stable liquid. This is shown in Fig. 8. Again, a curved diffusion path occurs, but this time it intersects the liquidus line in a metastable region (within the field of $\alpha_{2} \beta$ ). Dissolution of $\alpha$ occurs with the interface solution having the composition "H". Subsequent precipitation of $\alpha_{2} \beta$ causes the interface composition to change to a value near" $E$ ". Evidence for "incongruent dissolution" have been found and discussed ${ }^{(17)}$.

\section{Effect of Strain Energy}

Most phase diagrams show or infer the liquidus surfaces, $\ell$, as functions of composition and temperature, $\ell=\ell\left(T, x_{1}\right.$, $\mathrm{x}_{2} \ldots \mathrm{x}_{\mathrm{n}-1}$ at atmospheric pressure. The general lack of high pressure data is unfortunate, because oftentimes, we are 
concerned with dissolution at high pressure (i.e., within the earth, in meterorites or from the pressure created by surface forces on small particles, $\Delta \mathrm{P}=\sigma\left(1 / r_{1}+1 / r_{2}\right)$. The limitation can be diminished by using the following equation

$$
V_{m}\left(P-P_{0}\right)=R T \ln \left(X_{s a t}(P) / X_{\text {sat }}\left(P_{0}\right)\right)
$$

which related the effect of a pressure change $\left(P-P_{0}\right)$ on the solubility of a species of molar volume, $v_{m}$, into an ideal solution. If the solution is far from ideal activities rather than mol fractions must be used on the RHS of equation 5 . When the stress state is more complex than pure hydrostatic pressure, equation ' 5 must be modified further. If the strain energy density can be calculated, the effect on solubility is obtained through by substituting the strain energy density on the LHS of equation 5 .

Knowledge of the effect of a generalized stress on solubility is important to the understanding and control of stress corrosion. Stress corrosion in turn limits the practical strength of many glass and ceramic objects and may even be related to decay of $\operatorname{teeth}^{(18)}$.

Conclusions

Phase diagrams provide the best source of solubility data for high temperature systems and hence contribute decisively to prediction of dissolution behavior. Conversely, dissolution 
and crystallization studies are an effective means of locating liquidus surfaces accurately.

Acknow ledgement

Thanks are due to $A$. H. Heuer for his careful reading

of the manuscript and to N.A.S.A. Goddard space Flight Center for their support NGR 36-003-100. 
REFERENCES

1. A. A. Noyes and W. R. Whitney, Z. Physichal Chem., $23,689(1897)$.

2. W. Nernst, Z. Physichal Chem., 47, 52 (1904).

3. A. R. Cooper, Jr., J. Chem. Phys., 38, 1 (1963).

4. A. Berthoud, J. Chim. Phys., 10, 633 (1912).

5. a. E. A. Moelwyn Hughes, The KINETICS OF REACTIONS IN SOLUTION, Chpt. XII, Clarendon Press, Oxford, 1947.

b. L. Bircumshaw and A. Riddiford, Transport Controlled Processes, Quarterly Reviews, 6, 157 (1952).

c. W. D. Kingery, Corrosion of Refractories by Liquid Melts, p. 126-32 of ON HIGH TEMPERATURE - $\bar{A}$ TOOL FOR THE FUTURE, Stanford Research. Inst., Menlo Park, California (1956).

d. A. R. Cooper, Jr., Dissolution Kinetics in Glass Making, Advances in Glass Technology, Plenum Press, New York (1962).

6. The original suggestion was made by Prandtl:

I. Prandtl, Fluid Motion with Very Small Friction, Proc. III Intern. Math. Congr., Heidelberg (1904)

N.A.C.E., Tech. Memo No. 452 (1928).

A modern summary can be found:

V. G. Levich, Physicochemical Hydrodynamics, Prentice Hall (1962).

7. a. B. Levich, The Theory of Concentration Polarization, Dics. far. Soc., 1, 37 (1947).

b. D. G. Gregory, A. C. Riddiford, Transport to the Surface of a Rotating Disc, J.Chem.Soc., 1, 3756 (1956).

8. a. H. J. Tress, Some Distinctive Contours Worn on AluminaSilicate Refractory Faces by Different Molten Glasses: Surface Tension and the Mechanism of Refractory Attack, J. Soc. Glass Tech. , 38, 89 (1954).

b. c. $Y$. BOYS, SOAP BUBBIES AND THE FORCES WHICH MOULD THEM, Doubleday Anchor Books, Garden City, N.Y. (1959) • 
8. C. F. W. Preston and J. C. Turnbull, Physics of Upward Drilling, Am.J.Sci., 239, 92-105 (1941).

9. A. R. Cooper, Jr., The Use and Iimitations of the Concept of an Effective Binary Diffusion Coefficient for Multicomponent Systems, to be published in Trans. NBS Conference on Mass Transport, Oct. 1967.

10. A. R. Cooper, Jr., and W. D. Kingery, J.Am.Ceram.Soc., $47,37-43(1964)$.

11. I. Prigogine, Introduction to Thermodynamics of Irreversible Processes, Interscience, New York (1961).

12. F. C. Kracek, Carnegie Inst. Wash., Year Book Amm. Rep. Director Geophys. Lab. p.61-63 (1932-33) (Taken from Ref. 13, Fig. 365.)

13. E. M. Levin, C. R. Robbins and H. F. McMurdie, Phase Diagrams for Ceramists, The Am. Ceram. Soc., Columbus Ohio (1964).

14. Donald D. Fitts, Non Equilibrium Thermodynamics, Chpt. 8, McGraw-Hill Book Company, Inc. New York (1962).

15. a. J. S. Kirkaldy and I. C. Brown, Diffusion Behavior in Ternary Multiphase Systems, Can.Met.quart. 2 (I) $89-115$ (1963).

b. L, S. Darken, Diffusion of Carbon in Austenite With a Discontinuity in Composition, Trans. AIME, 180, 430 (1949).

C. F. N. Rhines, R. A. Meussner and R. T. DeHoff, Ternary Diffusion in Alpha Copper-Tin-Zinc, ibid, $212,860-62(1958)$.

16. a. A. R. Cooper, Jr., Physics \& Chemistry of Glasses, $6,55-61(1965)$.

b. T. O. Ziebold and A. R. Cooper, Jr., Acta Met., 13 $465-470$ (1965).

17. Y. Oishi, A. R. Cooper, Jr., and W. D, Kingery, Am.Ceram. Soc, 4 48, 88-95 (1965).

18. Speculation suggested by R. E. Ritter, University of Massachusetts, Amherst, Massachusetts. 
Fig. 1 Schematic of Concentration Distribution Near Interface Illustrating Definition of $\delta$ and $\delta$.

Fig. 2 Schenatic Showing Concentration Distribution Near Interface for (a) Transport Control, (b) Mixed Control, and (c) Phase Boundary Control.

Fig. 3 Silica Rich Portion of $\mathrm{Rb}_{2} \mathrm{O}-\mathrm{SiO}_{2}$ Phase Diagram Emphasizing Liquidus Lines.

Fig. 4 Plot of Log Rate of Disolution Versus Reciprocal Temperature. Sharp Curvature Suggests Inappropriateness of This Representation.

Fig. 5 Rate of Dissolution Versus Temperature (Schematic) For Case Where Transport Control Dissolution Kinetics and Phase Boundary Reaction Controls Precipitation Kinetics.

Fig. 6 Isothermal Liquidus Line and Diffusion Path for Dissolution of $\alpha$ in a solvent $S$ in the System $\alpha-\beta-\gamma$ ( - no compound formation - ).

Fig. 7 Concentration as a Function of Volume From Interface, $V$, for Dissolution of a Under Conditions Shown in Fig. 6. $V$ is Measured From Interface Following Direction of Gradient of $\alpha$.

Fig. 8 Isothermal Liquidus Lines and Diffusion Path in Hypothetical Ternary System $\alpha-\beta-\gamma$ for Case Where Compound, $\alpha_{2} \beta$, is Present at Specified Temperature. 
Fig. 1. Schematic of Concentration pistribution vear Interface Illustrating Definition of $\delta$ and $\delta$.

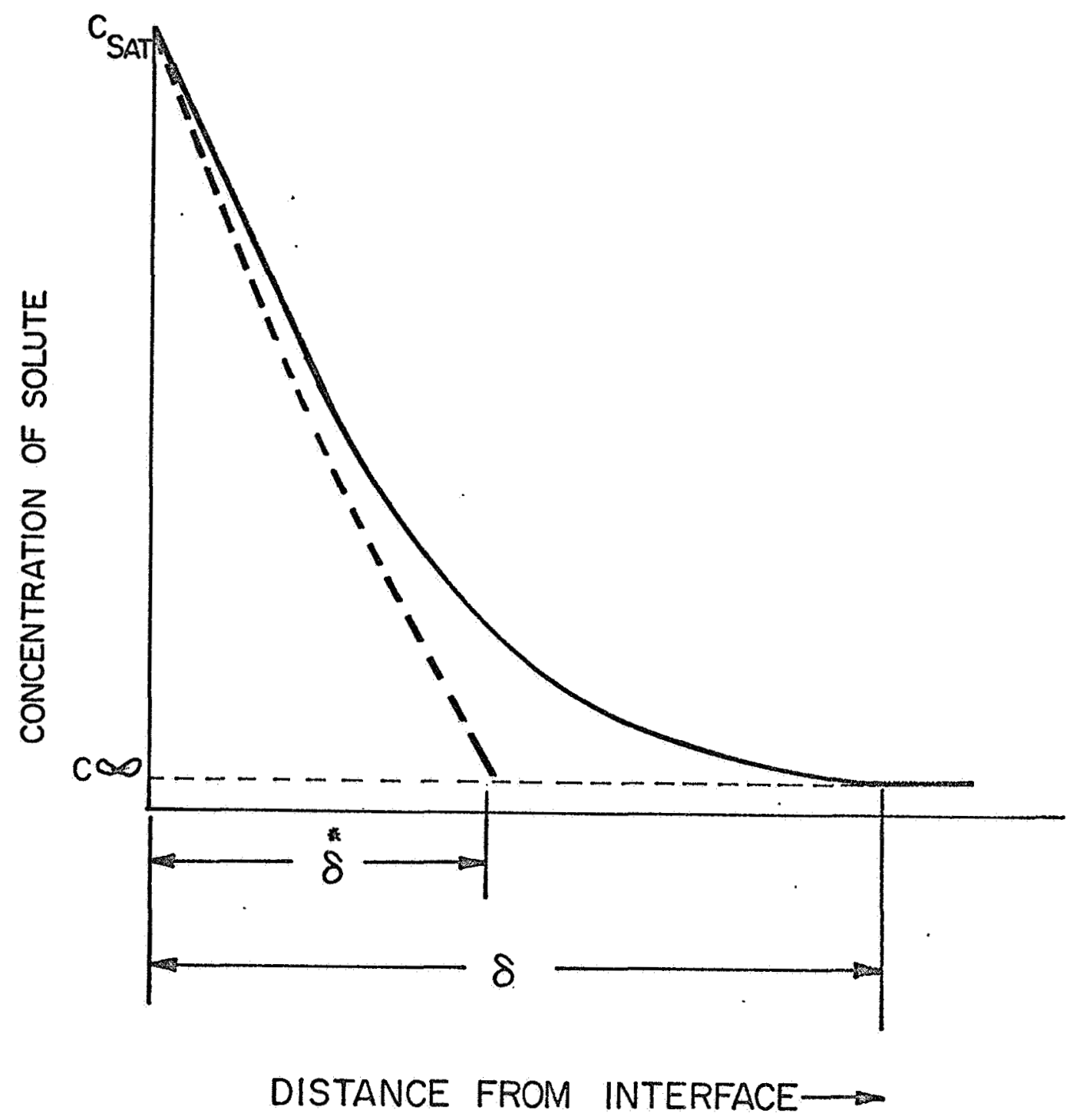

Fig 1 
Fig. 2 Schernatic Showing Concentration Distribution Near Interface for (a) Transport Control, (b) Mixed Control, and (c) phase Boundary control.

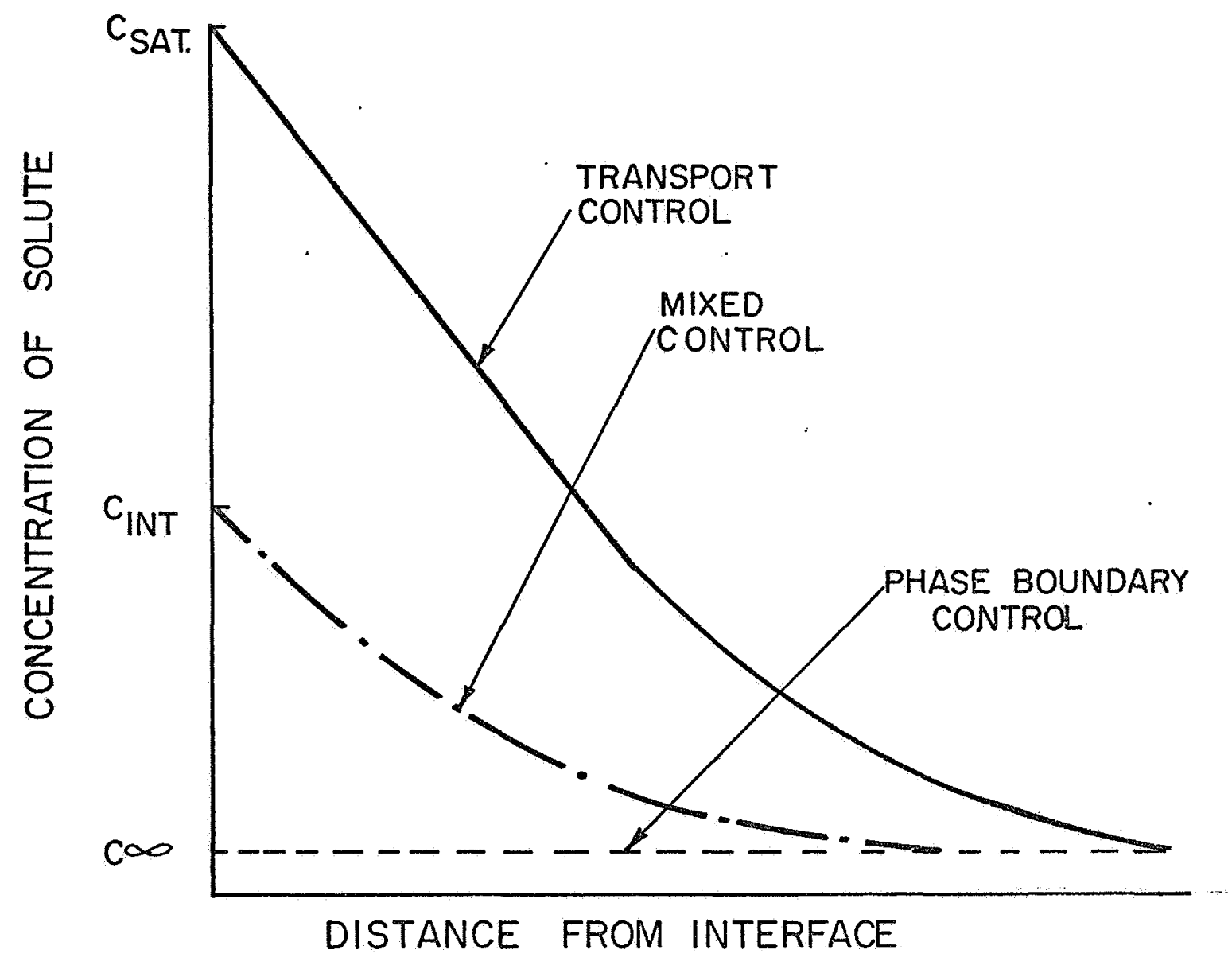

$$
\text { Fig } 2
$$


Fig. 3 Silica Rich portion of $\mathrm{Rb}_{2} \mathrm{O}-\mathrm{Si \textrm {O } _ { 2 }}$ Phase Diagram
Erphasizing Isiguidus Iines.

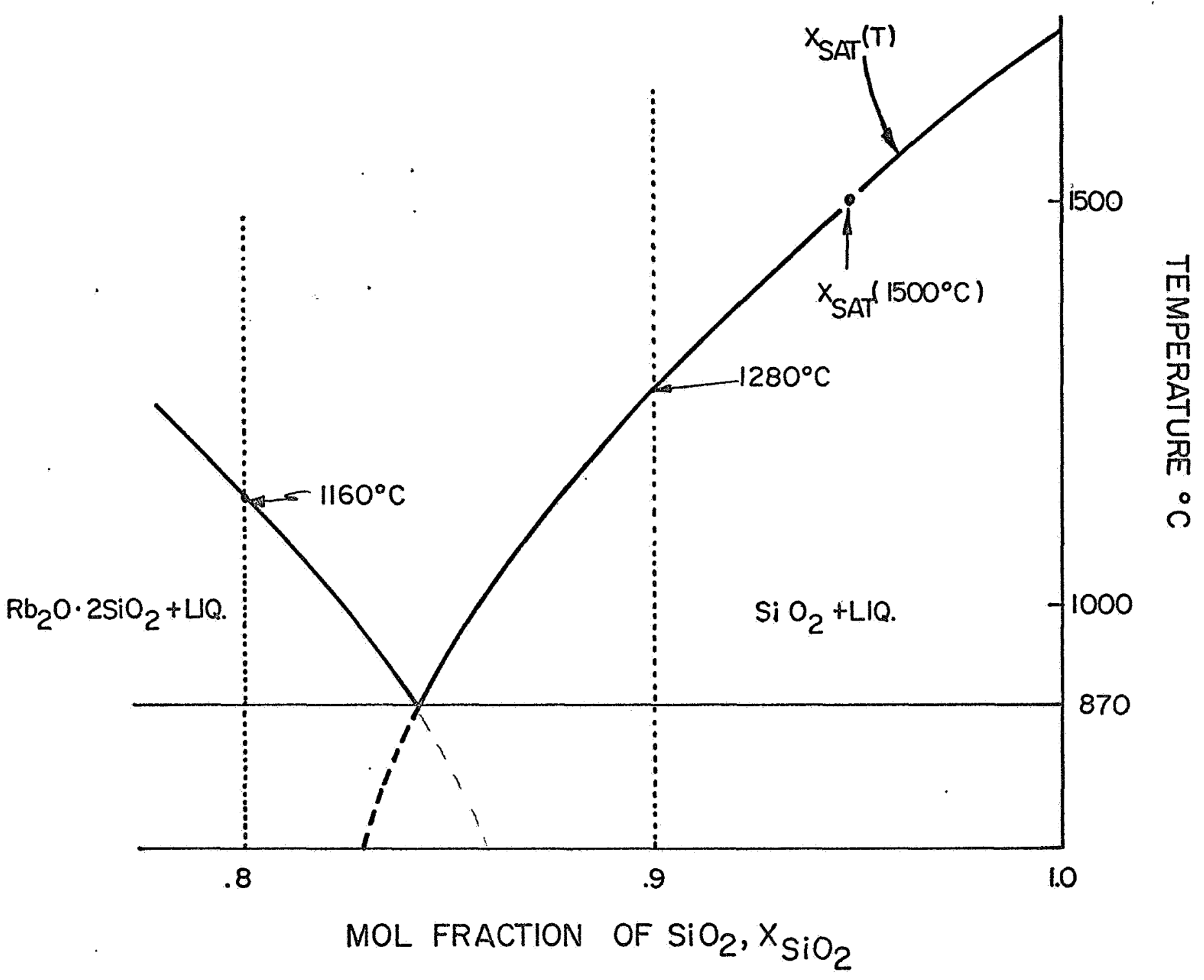

Fig 3 
$x$

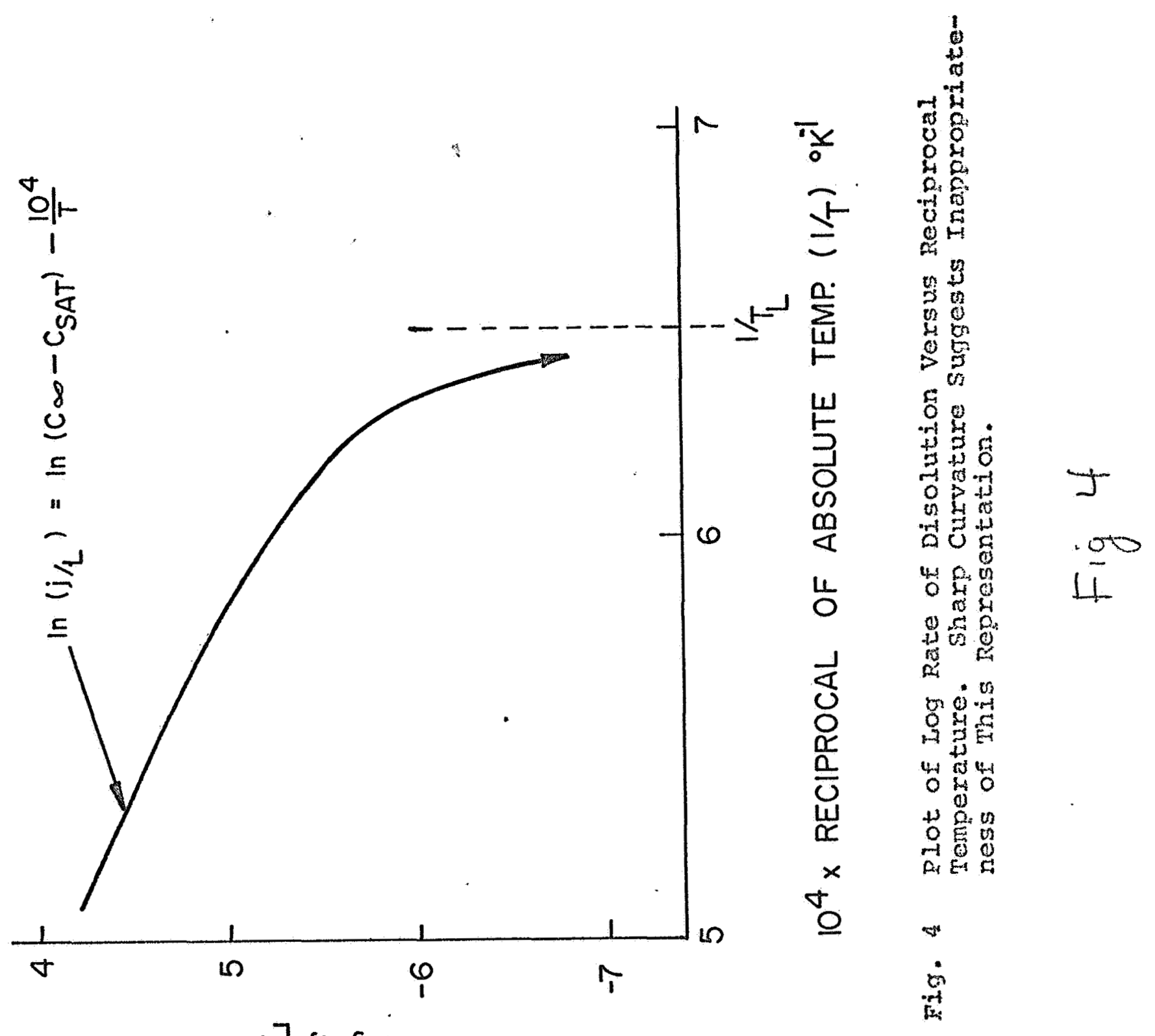

NOIIกาOSSIO 


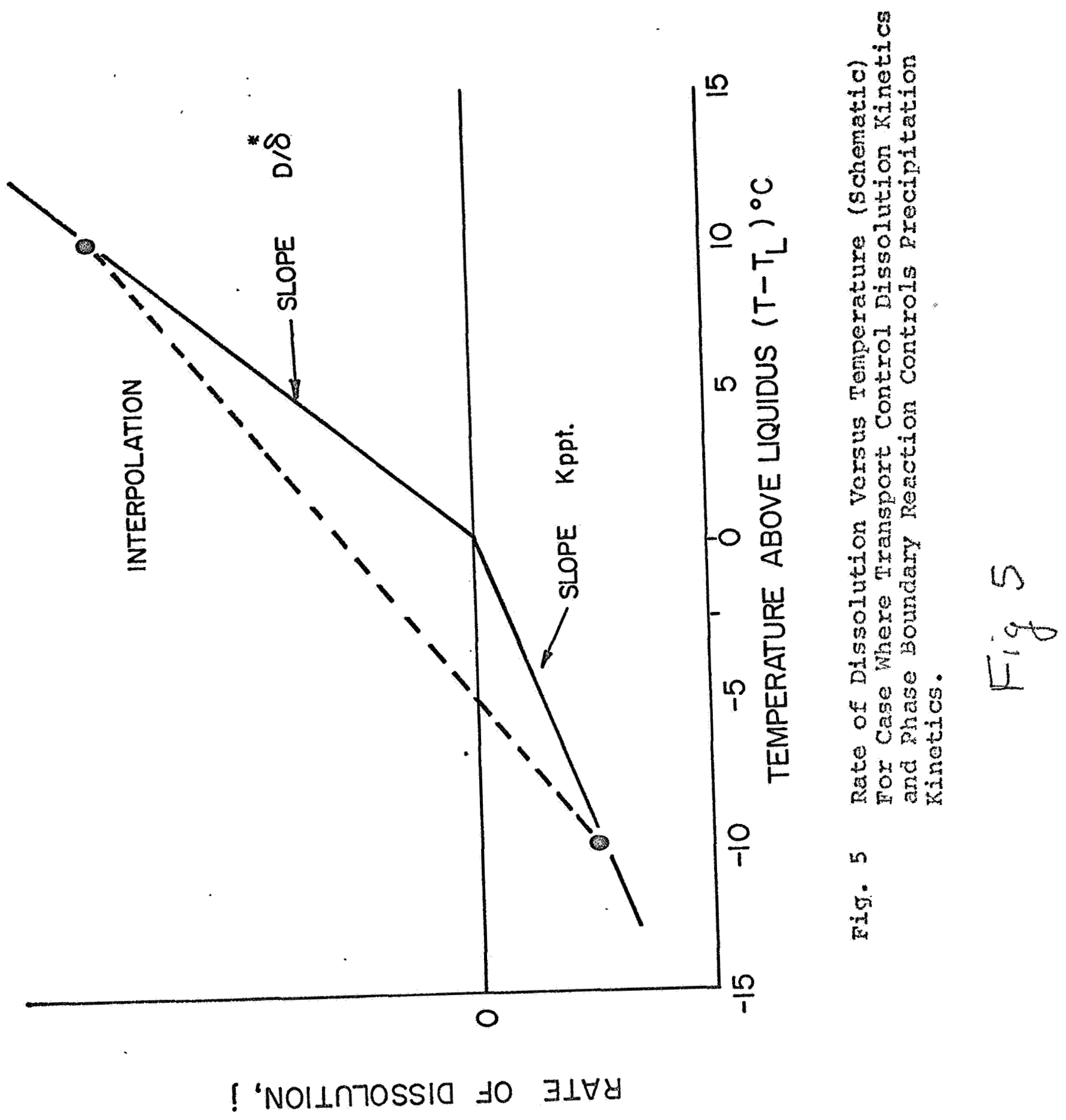




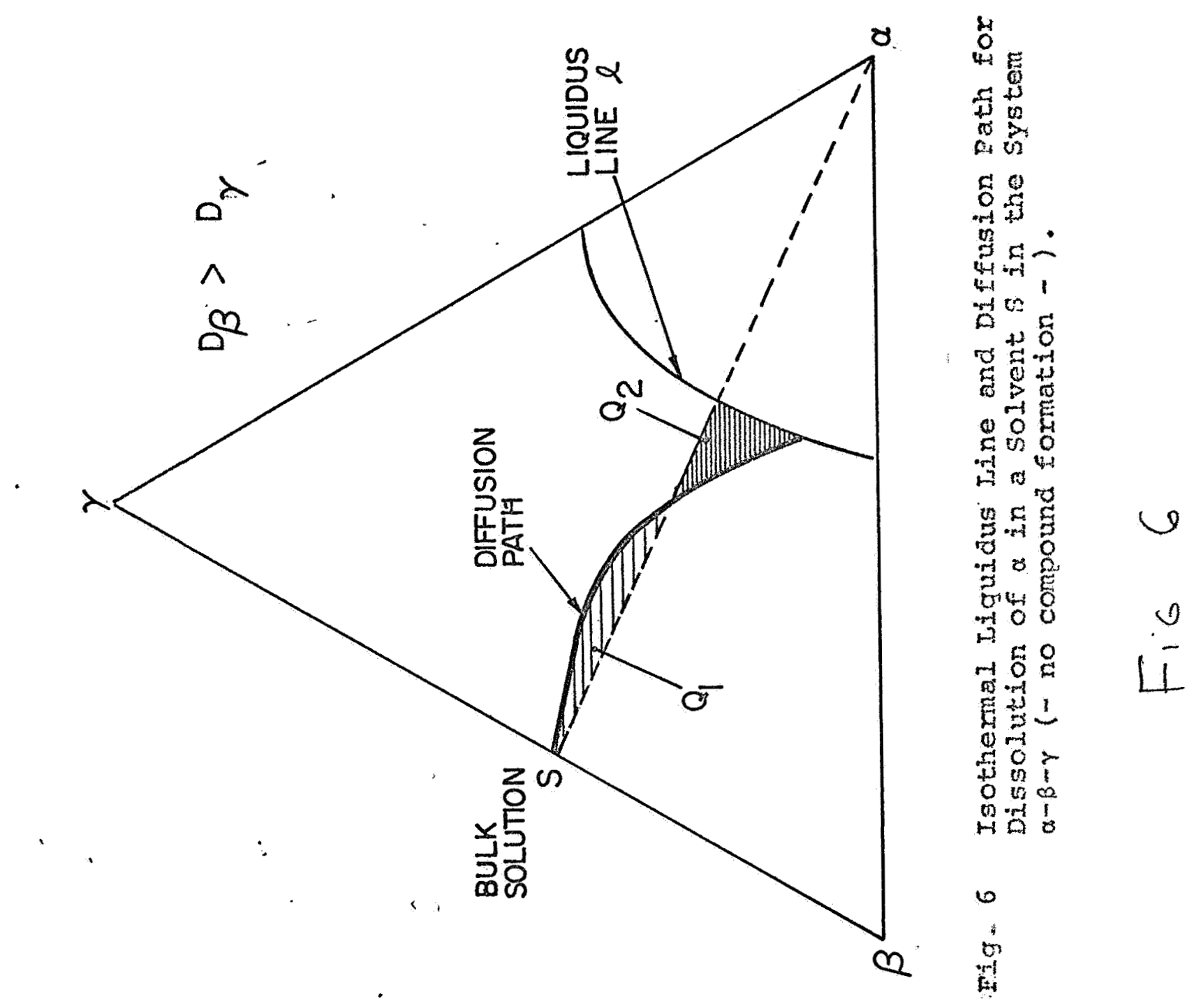


Fig. 7 Concentration as a Function of Volume From Interface, $V$, for Dissolution of $\alpha$ Under Conditions shown in Fig. 6. $V$ is Measured From Interface Following Direction of Gradient of $\alpha$.

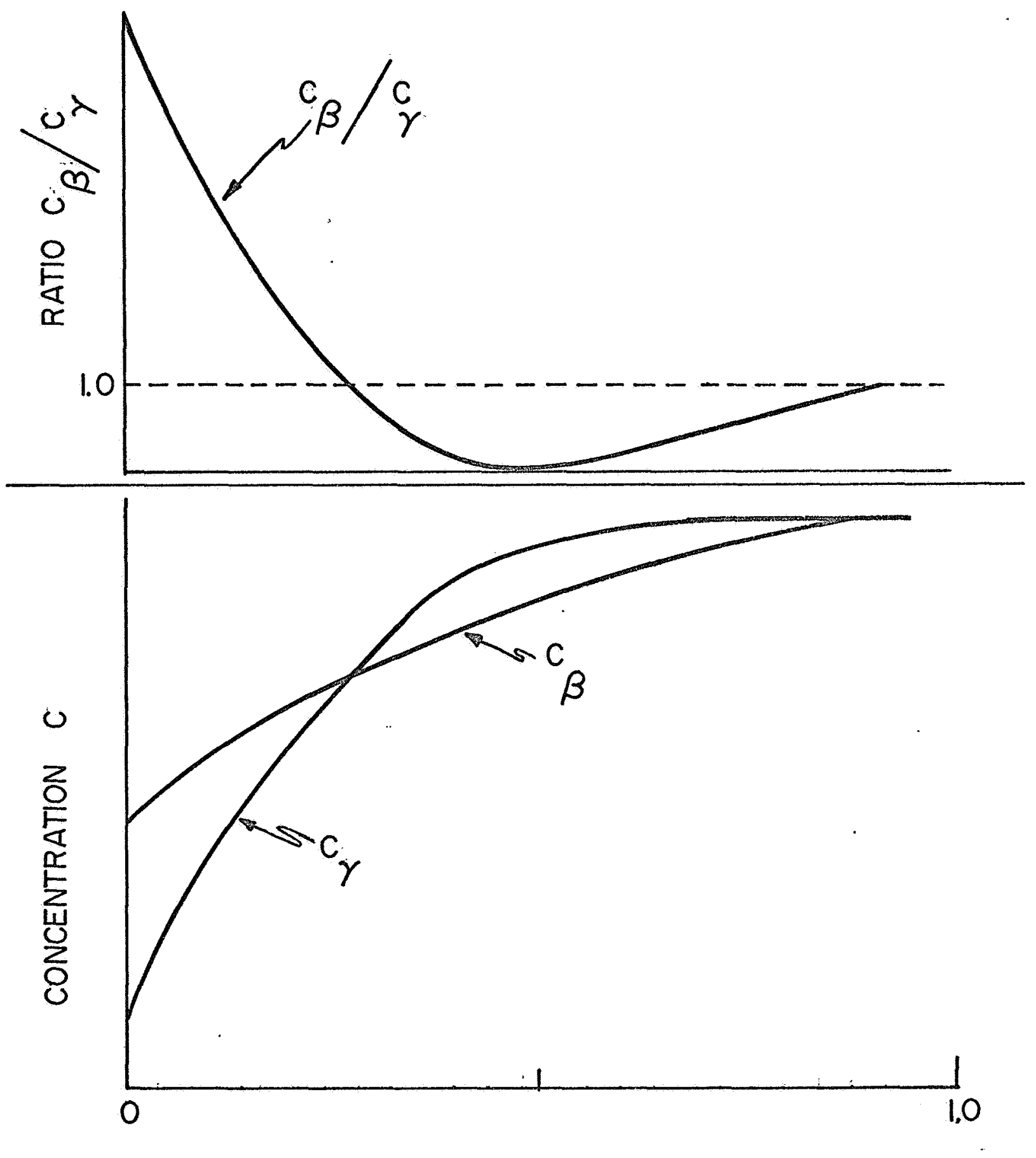

$$
\begin{gathered}
\text { VOLUME FROM INTERFACE, } \\
\text { TOTAL VOLUME OF SOLUTION, } \\
\text { FIg }
\end{gathered}
$$


Fig. B Isothermal Liquidus Lines and Diffusion Path in Hypothetical Texnaxy System $\alpha-\beta \rightarrow \gamma$ for Case where Compound, $\alpha_{2}{ }^{\beta}$, is Fresent at-Specified Temperature.

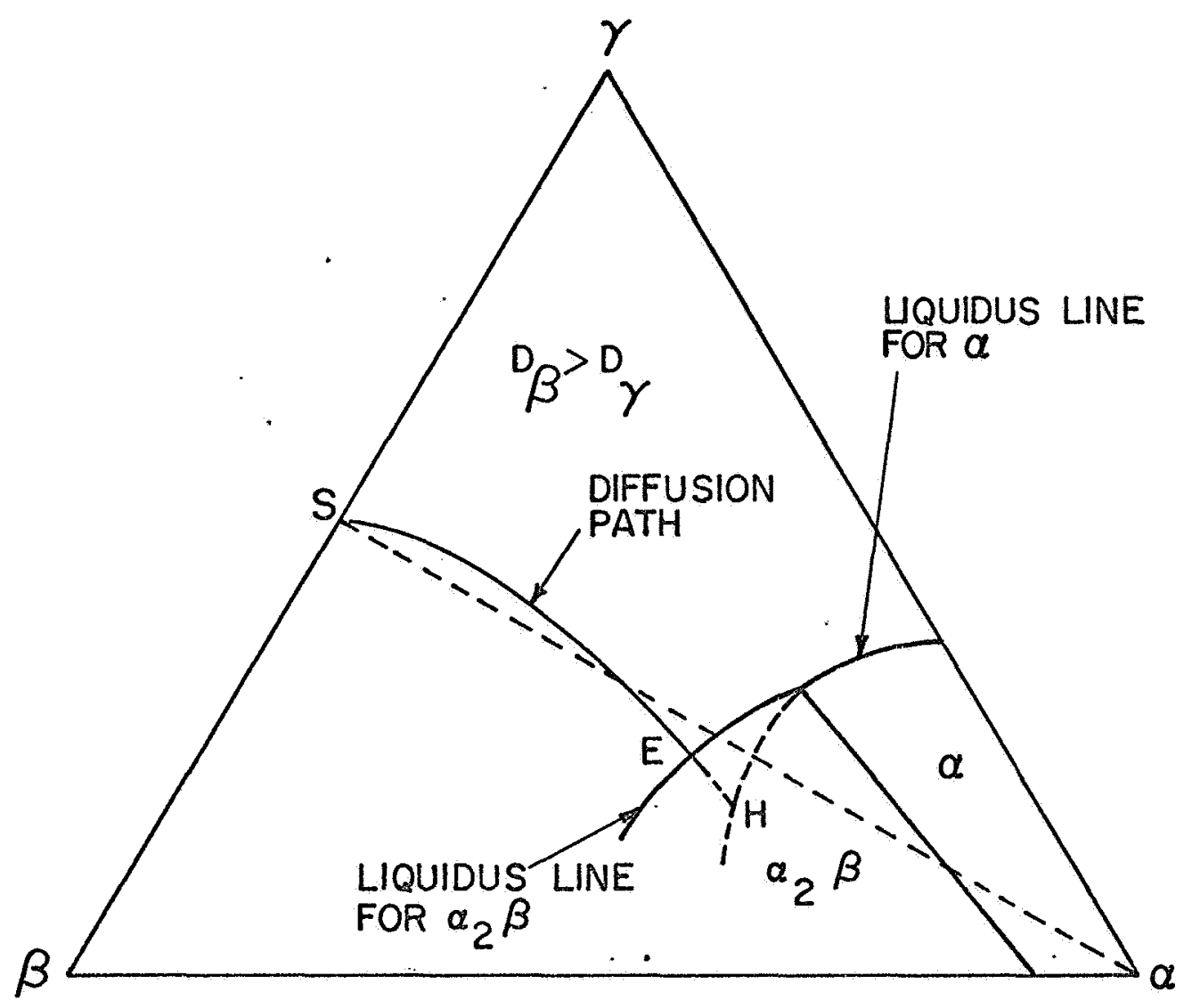

Fig 8 